\title{
The effect of beta-blockers on mortality in patients with heart failure and atrial fibrillation: A meta-analysis of observational cohort and randomized controlled studies
}

\author{
Gai-gai Ma ${ }^{1}$, Quan Fang ${ }^{2}$, Feng-xia Wang ${ }^{2}$ \\ ${ }^{1}$ Department of Cardiology, Peking Union Medical College Hospital, \\ Chinese Academy of Medical Sciences, Beijing, China \\ ${ }^{2}$ Department of Cardiology, People's Hospital of Xinjiang Uygur Autonomous Region, \\ Urumqi, Xinjiang, China
}

\begin{abstract}
Background: Beta-blockers (BB) are the cornerstone of therapy for heart failure (HF); however, the effects of these drugs on the prognosis of patients with concomitant atrial fibrillation $(A F)$ remain controversial. The objective of this meta-analysis was to evaluate the efficacy of BB on mortality in $H F$ coexisting with $A F$.

Methods: A systematic search of PubMed, Embase and the Cochrane Library databases was conducted. Observational cohort studies and randomized controlled trials reporting outcomes of mortality or $H F$ hospitalizations for patients with $H F$ and $A F$, being assigned to BB treatment. A non-BB group was also included.

Results: A total of 8 clinical studies ( 5 randomized controlled trials and 3 observational cohort studies) involving 34197 patients were included in the analysis. The pooled analysis demonstrated that $B B$ treatment was associated with a $22 \%$ reduction in relative risk of all-cause mortality in patients with $H F$ and $A F$ (RR: $0.78 ; 95 \%$ CI 0.71-0.86; $\left.p<0.00001 ; I^{2}=27 \%\right)$. The pooled analysis of 5 studies reported the outcome of $H F$ hospitalization (2774 patients) which showed that BB therapy was not associated with a reduction of $H F$ hospitalizations (RR: 0.94; 95\% CI 0.79-1.11; $p=0.46 ; I^{2}=38 \%$ ).

Conclusions: Meta-analysis suggests the potential mortality benefit of $B B$ in patients with $H F$ and $A F$. It was concluded herein that it is premature to deny patients with $A F$ and $H F$ to receive $B B$ therapy considering current evidence. (Cardiol J 2019; 26, 6: 744-752)
\end{abstract}

Key words: beta-blocker, atrial fibrillation, heart failure, mortality

\section{Introduction}

Heart failure (HF) and atrial fibrillation (AF) are two burdensome cardiovascular epidemics of the $21^{\text {st }}$ century [1-3]. Patients with concomitant $\mathrm{AF}$ and HF have even higher mortality and hospital admission rates [1-5]. Thus, the importance of concomitant $\mathrm{AF}$ and $\mathrm{HF}$ cannot be overstated.
Among the many therapies available for HF and $\mathrm{AF}$, beta-blockers (BB) are a cornerstone of management [5-8]. Based on several large randomized clinical trials, BB are strongly recommended (IA) for heart failure with reduced ejection fraction (HFrEF) by both American and European guidelines [5-8]. However, no randomized trials have been performed specifically to investigate the

Address for correspondence: Prof. Quan Fang, Department of Cardiology, Peking Union Medical College Hospital, Peking Union Medical College, Chinese Academy of Medical Sciences, No. 1 Shuaifuyuan, Wangfujing, Dongcheng District, Beijing, 100730, China, tel: +86-010-6529-5066, e-mail: 18800161900@163.com 
efficacy of BB in HF patients with AF. Post-hoc analyses of randomized trials designed to assess $\mathrm{BB}$ in $\mathrm{HF}$ suggest no benefit of $\mathrm{BB}$ in HF patients with AF [9-13]. Furthermore, two recent metaanalyses have failed to show clearly the mortality and morbidity benefit of $\mathrm{BB}$ in patients with $\mathrm{HF}$ and concomitant AF $[14,15]$. Of note, the AF group comprised only $17-21 \%$ of the whole patient cohort, and the obtained meta-analyzed results might reflect an under-powered analysis; in addition, the randomized controlled trials (RCTs) included in the previous meta-analyses were published more than 10 years ago, which was different from the current real world. Recently, several large well-designed observational cohort studies examining the prognostic effect of $\mathrm{BB}$ in $\mathrm{HF}$ and $\mathrm{AF}$ has been published after these metaanalyses were performed [16-18].

Given the limited evidence and uncertain effects of $\mathrm{BB}$ in $\mathrm{HF}$ with coexisting $\mathrm{AF}$, the aim was to conduct an updated meta-analysis of RCTs and observational cohort studies (OCSs) on the effect of $\mathrm{BB}$ on outcome in $\mathrm{HF}$ and $\mathrm{AF}$.

\section{Methods}

\section{Search strategy}

Electronic searches were conducted in the PubMed, Embase and the Cochrane Library databases. Search terms included "beta-blocker", "heart failure", "atrial fibrillation", and their variations. There was no language restriction placed on the searches. Each database was searched from inception to June 2017. Additionally, reference lists in the articles chosen for inclusion, and the reference lists of previous reviews were screened to identify other potentially eligible trials.

\section{Inclusion criteria}

Trials with the following characteristics were included:

- Population: Adult patients diagnosed as AF and $\mathrm{HF}$ (including both heart failure with reduced ejection fraction [HFrEF] and heart failure with preserved ejection fraction $[\mathrm{HFpEF}]$ ).

- Intervention: The intervention group included patients who received $\mathrm{BB}$ treatment.

- Control: The control group included patients who did not receive $\mathrm{BB}$ treatment.

- Outcomes: The all-cause mortality or HF hospitalizations had to be the outcome reported, and the duration of follow-up was at least 6 months.

- Types of study: The studies had to be RCTs or OCSs.

\section{Study selection and data extraction}

Two authors independently screened titles and abstracts. They obtained full articles that met the inclusion and exclusion criteria and after an independent review.

Information about the study and patient characteristics, methodological quality, intervention strategies, and clinical outcomes was systematically extracted separately by two reviewers. Disagreements were resolved by consensus.

\section{Quality assessment}

The quality of random control trial included was assessed by the Jadad quality scale [19]. The quality of the observational study was evaluated by the Newcastle-Ottawa Scale tool (available at: http://www.ohri.ca/programs/clinical_epidemiology/oxford.asp).

\section{Statistical analysis}

The relative risks (RRs) and 95\% confidence intervals (95\% CI) were used as the common measure across the studies. The hazard ratios (HRs) were considered equivalent to RRs [20, 21]. If the effect estimates were not available in the studies included, RRs were calculated by using the following formula: $\mathrm{RR}=$ Probability of events given treatment/Probability of events given no-treatment. If the studies provided the adjusted estimations, they were directly used in the meta-analysis. Statistical heterogeneity across studies was assessed by using the $\mathrm{Q}$ statistic with its $\mathrm{p}$ value and $\mathrm{I}^{2}$ statistic. The $\mathrm{I}^{2}$ statistic is used to quantify the proportion of total variation in the effect estimation that is due to between study variations. An $\mathrm{I}^{2}$ value greater than $50 \%$ indicates significant heterogeneity [22]. Clinical heterogeneity could not be excluded, so the pooled RR was calculated with the random-effects model [23].

\section{Results}

\section{Search and selection of studies}

The initial search yielded 680 unique titles and abstracts from PubMed, Embase and the Cochrane Library, and approximately potentially relevant articles identified. Of these articles, 5 RCTs [9-13] and 3 OCSs [16-18] fulfilled the eligibility criteria and were included in the present meta-analysis. The details of study selection flow diagram were described in Figure 1.

\section{Characteristics and quality of study included}

The characteristics of the studies included are presented in Table 1 . In all 8 studies included, 


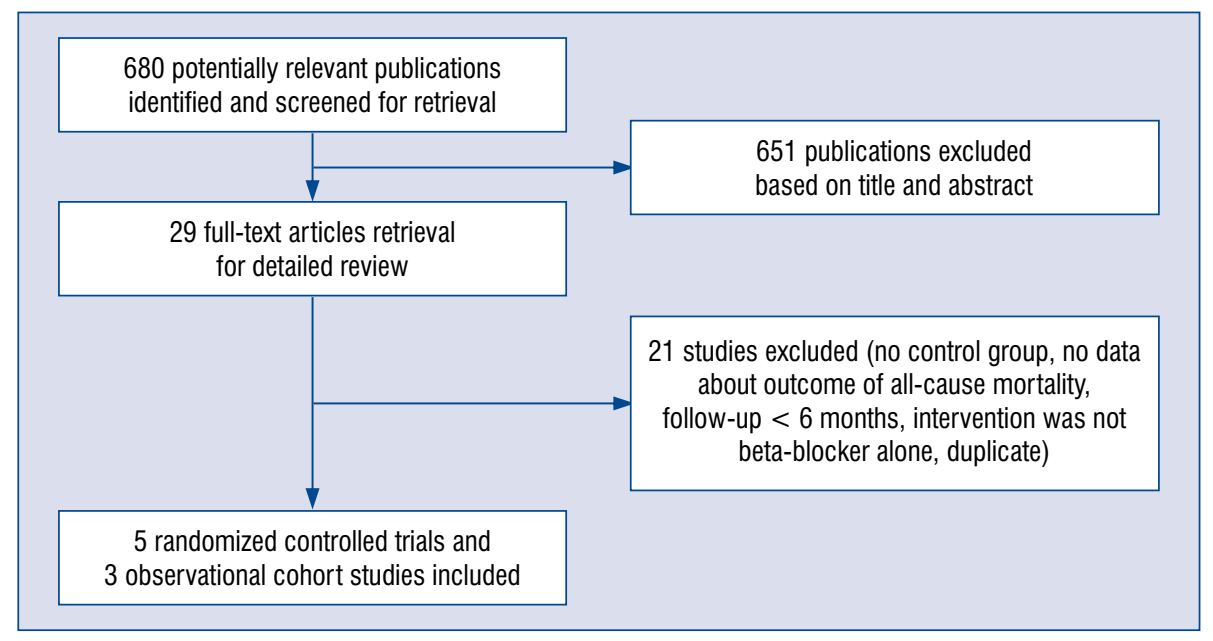

Figure 1. Selection process for articles included in meta-analysis.

5 studies were randomized controlled design and 3 studies were observational cohort design. All the $5 \mathrm{RCT}$ s were specific AF sub-studies of the large $\mathrm{HF}$ randomized trials that compared the effect of BB with those of placebo. Among the 3 OCSs, propensity score (PS) analysis was performed with PS matching in 2 studies (AF-CHF study and Danish nationwide registry), and multivariable-adjusted Cox regression analysis was performed in 1 study (Swedish HF Registry). Of the 8 studies, patients with HFrEF were included in the 6 studies (US-Carvedilol, CIBIS-II, MERIT-HF, BEST, Swedish HF Registry and AF-CHF); patients with both HFrEF and HFpEF were included in 2 studies (SENIORS and Danish nationwide registry). A total of 34197 patients were enrolled, including 20235 patients treated with BB and 13962 without BB. The mean followup duration ranged from 6 months to 3.1 years. For the 5 RCTs, study quality was scored as "good" for all but one (the US-Carveilol study), which was scored as "fair" by using the Jadad quality scale. For the 3 OCSs, study quality was scored as good (7-9 scores) by using Newcastle-Ottawa Scale tool.

\section{Patient characteristics}

Patient characteristics of the studies included are presented in Table 2 . Included patients were a mean age of 70 years, $76 \%$ were men, mean left ventricular ejection fraction was $27.5 \%, 35 \%$ had New York Heart Association (NYHA) functional class for I/II and 65\% had NYHA III/IV. Coronary artery disease was common and ranged from $21 \%$ to $56 \%$, respectively; hypertension and diabetes were also common and ranged from $8 \%$ to $56 \%$ and from $13 \%$ to $27 \%$, respectively. Baseline medication included angiotensin converting enzyme inhibitor/angiotensin receptor blocker in $84.8 \%$ of the patients, digoxin in $70 \%$, diuretics in $90 \%$ and oral anticoagulant in $60 \%$. Baseline heart rate of patients was similar among included studies, ranging from $79 \mathrm{bpm}$ to $88 \mathrm{bpm}$. $5 \mathrm{RCTs}$ reported the heart rate change at the end of follow-up with a mean heart rate reduction of $10.9 \mathrm{bpm}$.

\section{Effect of beta-blockers on all-cause mortality}

All 8 studies reported the outcome of all-cause mortality. The effect estimations of HRs were provided in 5 studies and RRs in 3 studies. The effect of $\mathrm{BB}$ on all-cause mortality in $\mathrm{HF}$ and $\mathrm{AF}$ was shown in Figure 2. In the pooled analysis of 5 RCTs, $\mathrm{BB}$ use was associated with non-significant reduced risk for mortality (RR 0.97 ; 95\% CI 0.79-1.19, $\mathrm{p}=0.79$; heterogeneity, $\mathrm{p}=0.55, \mathrm{I}^{2}=0$ ). In the pooled analysis of 3 OCSs, BB use was associated with improved survival (RR 0.74; 95\% CI 0.71-0.78, $\mathrm{p}<0.00001$; heterogeneity, $\left.\mathrm{p}=0.22, \mathrm{I}^{2}=0\right)$. Overall, use of $\mathrm{BB}$ reduced risk for mortality by $22 \%$ (RR $0.78 ; 95 \%$ CI 0.71-0.86, $\mathrm{p}<0.00001$; heterogeneity, $\left.\mathrm{p}=0.26, \mathrm{I}^{2}=27 \%\right)$.

\section{Effect of BB on HF hospitalization}

Five studies reported the outcome of HF hospitalization (CIBIS-II, MERIT-HF, SENIORS, BEST and AF-CHF), including 2774 patients. The pooled analysis showed that BB therapy was not associated with a reduction of $\mathrm{HF}$ hospitalizations (RR 0.94; 95\% CI 0.79-1.11, $\mathrm{p}=0.46$; heterogeneity, $\mathrm{p}=0.17, \mathrm{I}^{2}=38 \%$ (Fig. 3 ). 


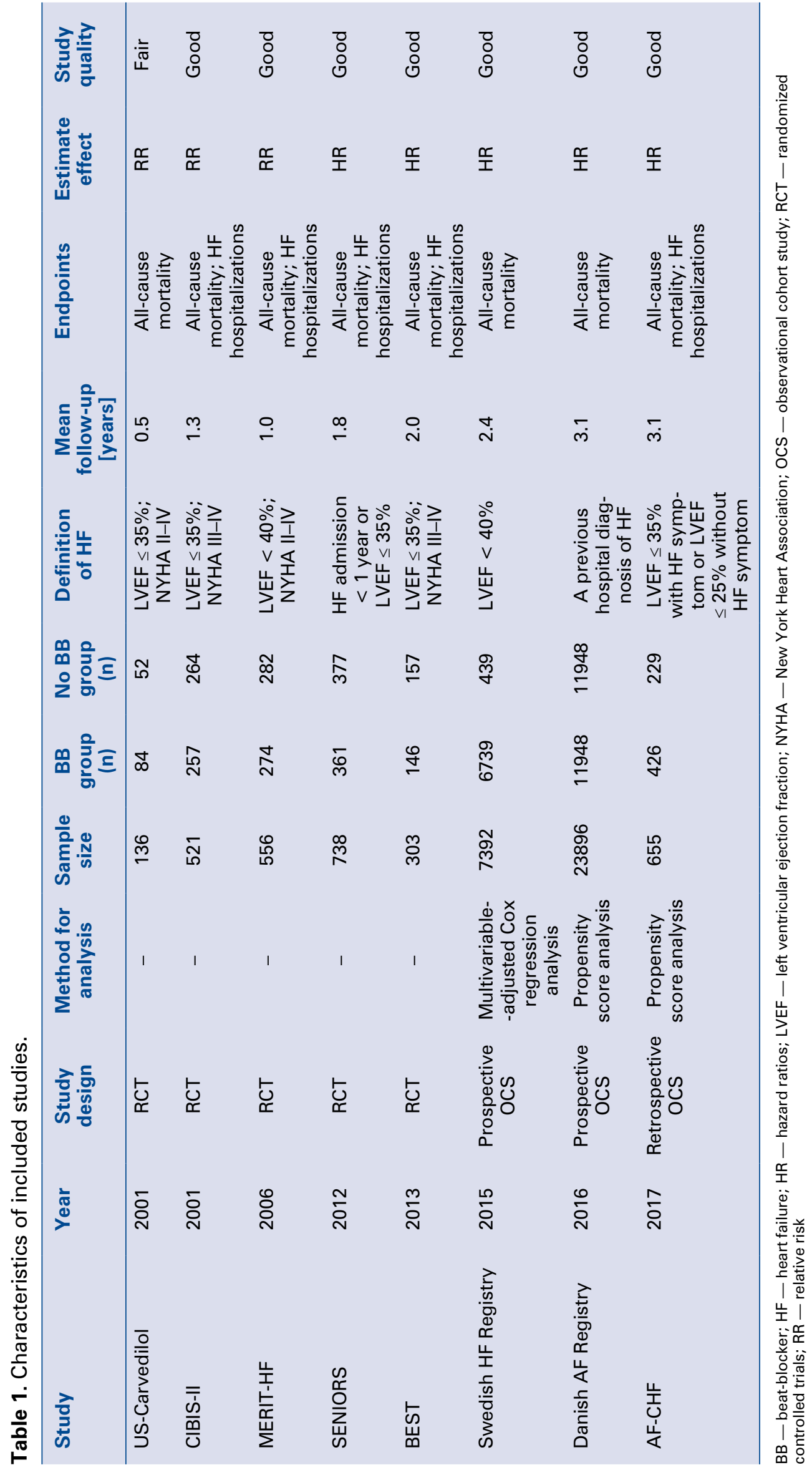




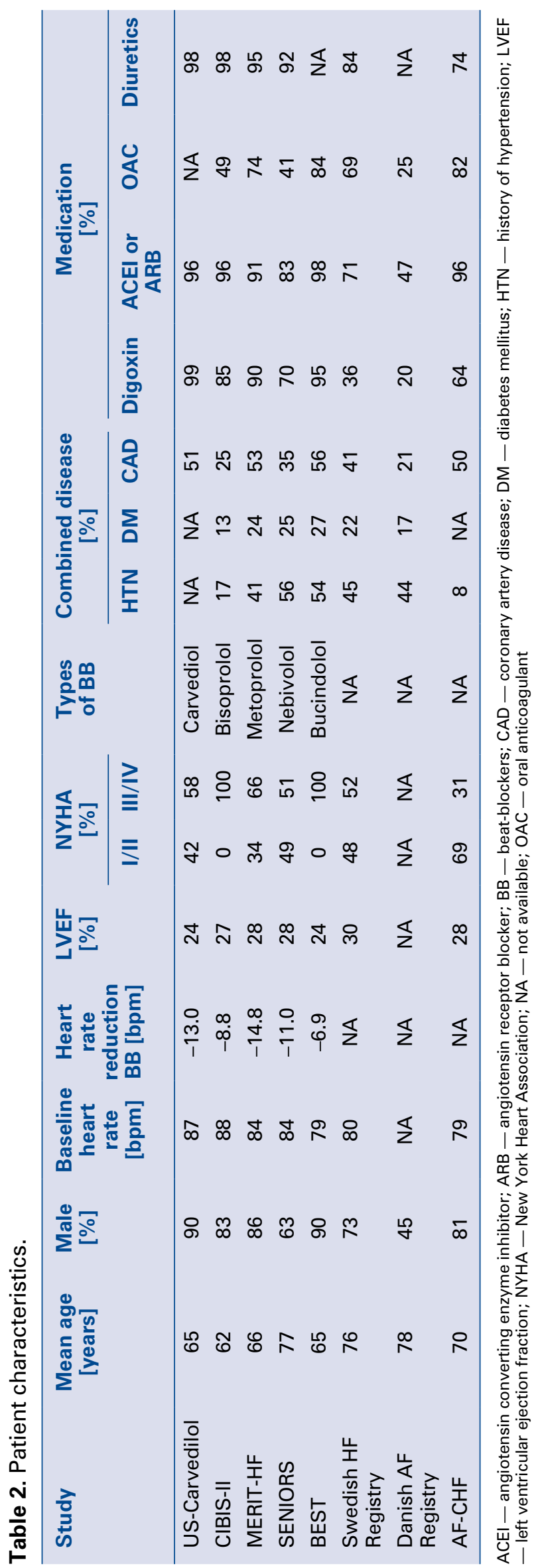

\section{Sensitivity analysis}

Leave-one-out sensitivity analysis on all-cause mortality was performed by omitting one study at a time, and found that none of the individual studies significantly influenced the pooled estimate of all-cause mortality. Subgroup analyses showed that when the pooled analysis of all-cause mortality was performed using fixed-effect model, a similar result was observed.

\section{Discussion}

\section{Main findings}

Meta-analysis of 8 studies involving 34197 patients revealed that $\mathrm{BB}$ were associated with a $22 \%$ reduction in all-cause mortality. Although the finding was limited to observational studies and trends favored $\mathrm{BB}$ for $\mathrm{HF}$ hospitalization did not reach statistical significance. Overall, results supported current evidence-based recommendations to pursue $\mathrm{BB}$ in all $\mathrm{HF}$ patients with or without $\mathrm{AF}$.

\section{Comparison with other studies}

The present observed mortality benefit of $\mathrm{BB}$ in $\mathrm{HF}$ and $\mathrm{AF}$ diverged from two earlier metaanalyses $[14,15]$. The latest systematic review conducted by Kotecha et al. [15] was more recent and comprehensive, which was an individual patient-level meta-analysis. However, in the 2 earlier meta-analyses, only RCTs were included and the AF group comprised only $17-21 \%$ of the whole patient cohort. Therefore, the obtained metaanalyzed results might reflect an under-powered analysis. In meta-analysis, the number of patients in the included 5 RCTs was 2254 with 407 deathevents, which is still low for survival analysis, and the possibility that a lack of power may have played a role could not be excluded. Additionally, it is important to recognize that the included $5 \mathrm{RCT}$ s were not specifically designed to assess the effect of $\mathrm{BB}$ in patients with $\mathrm{AF}$ and HF. Above all, the benefits of $\mathrm{BB}$ for all-cause mortality or HF hospitalizations were not observed in the pooled analysis of $5 \mathrm{RCTs}$, consistent with the earlier meta-analyses.

Our meta-analysis included recently published 3 observational studies with a low heterogeneity. The 3 observational studies from large registries included were well designed by using PS analysis and multivariable-adjusted Cox regression analysis to reduce the effects of confounders (including age, sex, underlying disease, medications, and NYHA functional class). The mortality benefit associated with $\mathrm{BB}$ in this analysis was largely driven by the results of Danish AF Registry [15]. However, the 


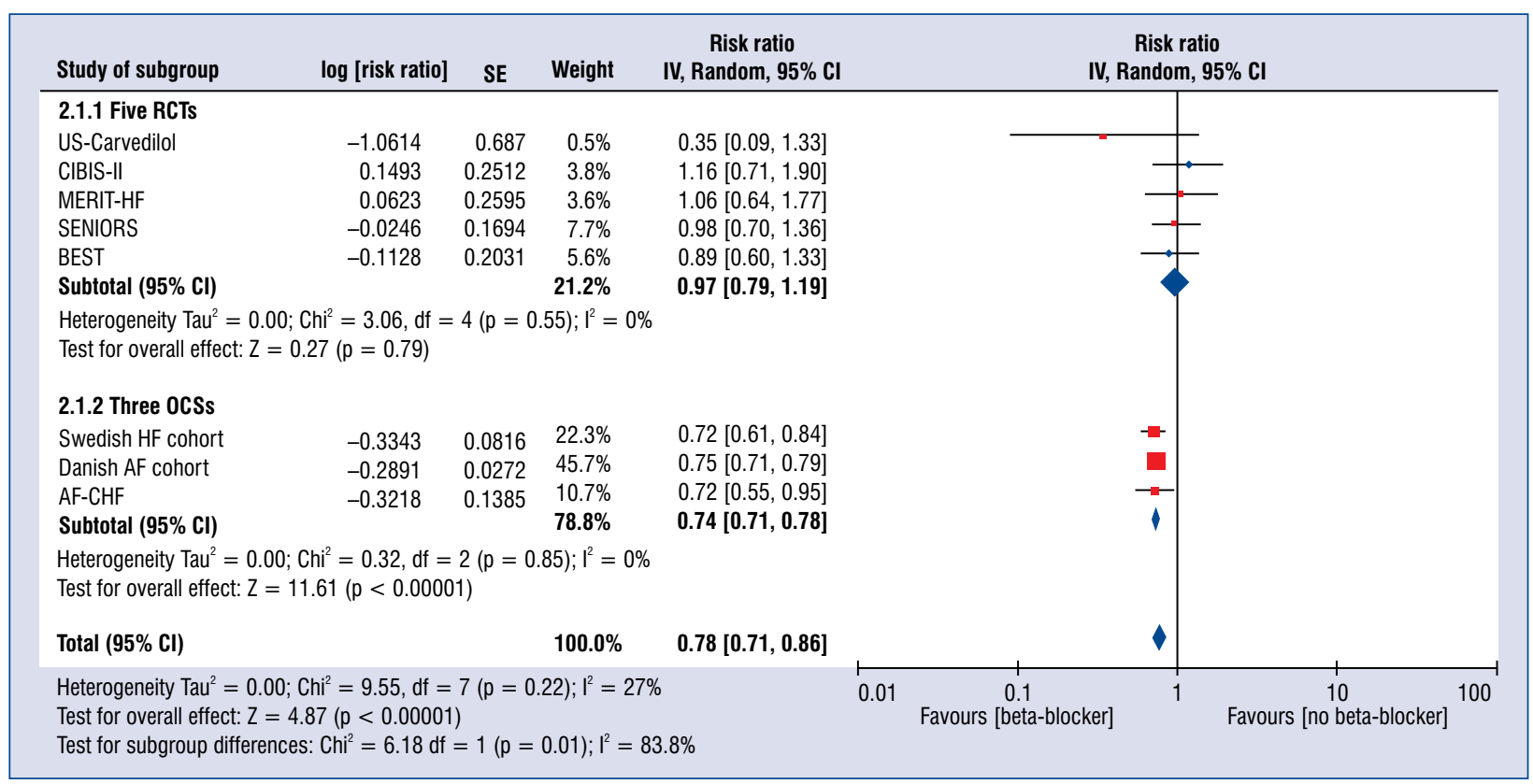

Figure 2. Effect of beta-blockers on all-cause mortality; $\mathrm{Cl}$ - confidence interval; OCSs - observational cohort study; RCTs - randomized controlled trials.

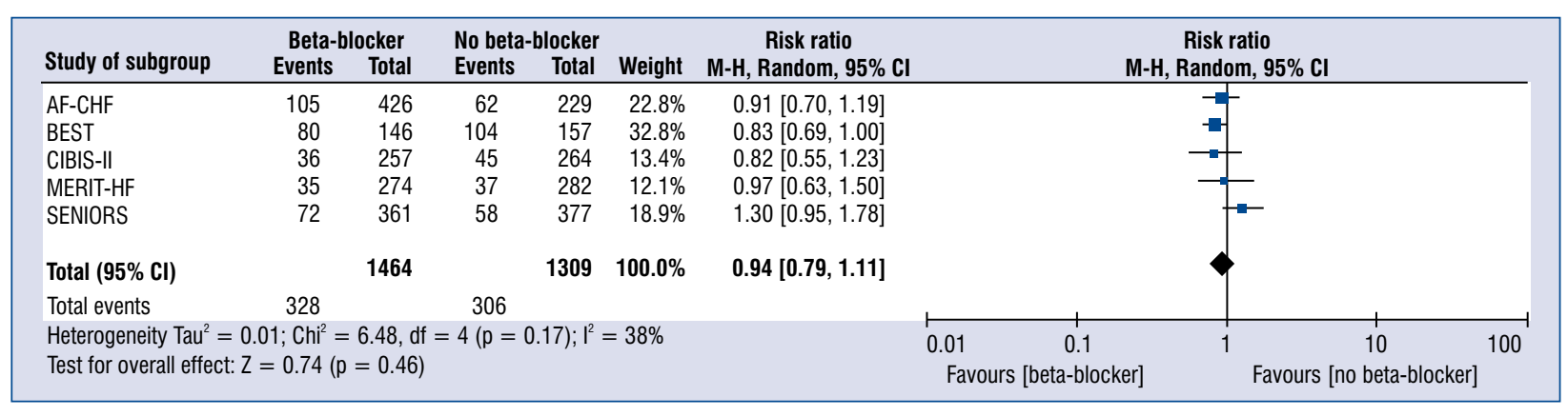

Figure 3. Effect of beta-blockers on heart failure hospitalization.

protective effect still remained after removing this study using the sensitivity analysis. Furthermore, both fixed and random effect models in the pooled analysis shows the significantly similar benefit of $\mathrm{BB}$ treatment. Accordingly, the conclusion that the treatment of $\mathrm{BB}$ reduces all-cause mortality in patients with $\mathrm{HF}$ and $\mathrm{AF}$ is fairly reliable.

It must be noted the difference between outcomes in included 5 RCTs and 3 OCSs. The present finding that $\mathrm{BB}$ therapy decreased mortality in patients with $\mathrm{HF}$ and $\mathrm{AF}$ was limited to the $3 \mathrm{ob}-$ servational studies. The controversial results may be partly explained by differences in methodology, patient demographics, HF severity, concomitant medication or follow-up duration. First, baseline characteristics of patients differed in these studies. In 5 RCTs, almost all patients included were symptomatic HF with NYHA II-IV, while in observational studies asymptomatic HF with NYHA I were also included. It seems that patients included in OCSs were at lower risk and had better BB tolerance, which all factors associated with lower mortality. Second, combined treatment was also an important confounder. In $5 \mathrm{RCTs}$, patients with $\mathrm{HF}$ and $\mathrm{AF}$ were more commonly treated with digoxin (88\%), while in 3 OCSs, only $40 \%$ of patients were treated with digoxin. Digoxin had been reported to be associated with increased mortality in $\mathrm{AF}$ patients. The potential synthetic adverse effect of digoxin cannot be completely eliminated. Another possible reason as mentioned before was that the small number of patients in the included 5 RCTs was 2254 with 407 death-events which might reflect an under-powered analysis. It was admitted 
that a well-designed randomized trial would be of great value according to the highest standards of evidence-based medicine. In RCTs the BB were well defined, with determined type and dose of $\mathrm{BB}$ and also heart rate reduction during therapy. While observational studies had inherent limitations including nonuniformly defined variables across studies. The use of BB differed between studies with a different type, dose and course. Based on observational studies, whether the doses and types of $\mathrm{BB}$ affect the effects of $\mathrm{BB}$ in patients with $\mathrm{HF}$ and $\mathrm{AF}$ could not be assessed. Until more solid evidence is available, it is premature to deny patients with $\mathrm{AF}$ and $\mathrm{HF} \mathrm{BB}$ therapy considering current evidence.

\section{Possible mechanisms for findings}

The optimal heart rate target in AF patients is unclear. Moreover, there is limited evidence for lenient rate control for AF patients with $\mathrm{HF}$. Previous studies have mainly examined sinus rhythm, and whether a higher heart rate is associated with worse outcomes in HF with concomitant $\mathrm{AF}$ has not been adequately studied. The lack of a relationship between heart rate and outcomes in patients with $\mathrm{HFrEF}$ and concomitant $\mathrm{AF}$ has previously been described [24-27]. In Rate Control Efficacy in Permanent Atrial Fibrillation: A Comparison between Lenient versus Strict Rate Control II (RACE II) trial, lenient rate control $(<110 \mathrm{bpm})$ did not yield worse outcomes than strict rate control $(<80 \mathrm{bpm})$ overall or in the subgroup of patients with $\mathrm{HF}[28,29]$. Potential mortality benefit of $\mathrm{BB}$ in $\mathrm{HF}$ coexisting with $\mathrm{AF}$ has been observed in the present meta-analysis. However, this meta-analysis does not provide the possible mechanisms for the survival benefit of BB in those patients without specific initial data on heart rate and heart rate change during $\mathrm{BB}$ treatment. The Swedish HF Registry [18] included in the present meta-analysis is the only study to assess an association of heart rate strata and $\mathrm{BB}$ use with all-cause mortality in patients with $\mathrm{HF}$ in AF. The study showed that higher resting heart rate was associated with increased mortality in AF, which was true only if heart rate $>100 \mathrm{bpm}$. Furthermore, $\mathrm{BB}$ use was associated with reduced mortality in patients with $\mathrm{AF}$, and a lower heart rate was associated with reduced mortality in $\mathrm{AF}$ only for those with heart rate $\leq 100 \mathrm{bpm}$. In our meta-analysis, baseline heart rate of patients were similar among included studies, ranging from $79 \mathrm{bpm}$ to $88 \mathrm{bpm}$ ( $<100 \mathrm{bpm}$ ); only $5 \mathrm{RCTs}$ had reported the heart rate change for $\mathrm{BB}$ treatment at the end of follow-up, with a mean heart rate reduction of $10.9 \mathrm{bpm}$. However, in the pooled analysis of $5 \mathrm{RCTs}$, BB use was associated with non-significant reduced risk for mortality. Except for small sample size of an under-powered analysis, another possible explanation is that patients included in the trials benefit less from $\mathrm{BB}$ use with a baseline heart rate $<100 \mathrm{bpm}$. Patients with a higher heart rate may possibly benefit from BB treatment according to the results of Swedish HF Registry.

\section{Implications for clinical practice}

Considering the current controversies and challenges, more studies on BB in patients with $\mathrm{HF}$ and $\mathrm{AF}$ are still needed. Randomized controlled trials on $\mathrm{BB}$ for $\mathrm{HF}$ with concomitant $\mathrm{AF}$ may not be feasible because of ethical reasons. Thus, well designed and analyzed cohort studies from large registry will be more expected, which can give us more information from the real world. Future investigation should also help determine which patients with $\mathrm{AF}$ and $\mathrm{HF}$ will derive the greatest benefit from $\mathrm{BB}$ therapy, including those with $\mathrm{HFrEF}$ or HFpEF, older or younger, baseline heart rate. Additionally, the potential benefit of $\mathrm{BB}$ and their potential mechanisms beyond $\mathrm{HR}$ reduction in $\mathrm{HF}$ coexisted with $\mathrm{AF}$ also require further study.

\section{Limitations of the study}

The present analysis has several limitations that must be taken into consideration when interpreting the results. First, observational studies were included in the analysis and the mortality benefit was largely driven by those OCSs. Because of the observational nature of the cohort study and lack of randomization, the effect of unmeasured or residual confounding could not be ruled out. Although 3 observational studies from large registries were well designed by using PS analysis and multivariate regression analysis to reduce the effects of confounders, it would be specially mentioned that not all the studies adjusted for all covariates, so combined results should be interpreted with caution. Even though a very low heterogeneity was showed in the present analysis, clinical heterogeneity could not be underestimated. Therefore, a random-effect model was used in the meta-analysis and sensitivity analysis was also used to explore possible study characteristics that might have influenced the pooled estimates. Inherent limitations of pooled analysis of studies include the limited availability of confounding variables, including the type and dose of $\mathrm{BB}$, the course of 
treatment. Also, in the present analysis, the effects of different BB therapies were pooled and thereby assumed a class effect. However, specific differences in pharmacologic profiles may have added to the heterogeneity of the cohort and thereby the results. Finally, this analysis pooled study group estimates and did not assess individual patient data, which limits the possibility of adjustment for individual patient characteristics.

\section{Conclusions}

In summary, the present meta-analysis suggested the potential mortality benefit of $\mathrm{BB}$ in $\mathrm{HF}$ coexisting with $\mathrm{AF}$, and supported current evidence-based recommendations to pursue $\mathrm{BB}$ for those patients. It was concluded that it is premature to deny patients with $\mathrm{AF}$ and $\mathrm{HF}$ beta-blocker therapy considering current evidence.

\section{Conflict of interest: None declared}

\section{References}

1. Benjamin EJ, Blaha MJ, Chiuve SE, et al. American Heart Association Statistics Committee and Stroke Statistics Subcommittee. Heart Disease and Stroke Statistics-2017 Update: A Report From the American Heart Association. Circulation. 2017; 135(10): e146-e603, doi: 10.1161/CIR.0000000000000485, indexed in Pubmed: 28122885.

2. Heidenreich PA, Albert NM, Allen LA, et al. Forecasting the impact of heart failure in the United States: a policy statement from the American Heart Association. Circ Heart Fail. 2013; 6(3): 606-619, doi: 10.1161/HHF.0b013e318291329a, indexed in Pubmed: 23616602.

3. Krijthe BP, Kunst A, Benjamin EJ, et al. Projections on the number of individuals with atrial fibrillation in the European Union, from 2000 to 2060. Eur Heart J. 2013; 34(35): 2746-2751, doi: 10.1093/eurheartj/eht280, indexed in Pubmed: 23900699.

4. Olsson LG, Swedberg K, Ducharme A, et al. Atrial fibrillation and risk of clinical events in chronic heart failure with and without left ventricular systolic dysfunction: results from the Candesartan in Heart failure-Assessment of Reduction in Mortality and morbidity (CHARM) program. J Am Coll Cardiol. 2006; 47(10): 1997-2004, doi: 10.1016/j.jacc.2006.01.060, indexed in Pubmed: 16697316.

5. January CT, Wann LS, Alpert JS, et al. 2014 AHA/ACC/HRS guideline for the management of patients with atrial fibrillation: executive summary: a report of the American College of Cardiology/American Heart Association Task Force on practice guidelines and the Heart Rhythm Society. Circulation. 2014; 130(23): 2071-2104, doi: 10.1161/CIR.0000000000000040, indexed in Pubmed: 24682348.

6. Kirchhof P, Benussi S, Kotecha D, et al. 2016 ESC Guidelines for the management of atrial fibrillation developed in collaboration with EACTS. Eur Heart J. 2016; 37(38): 2893-2962, doi: 10.1093/eurheartj/ehw210, indexed in Pubmed: 27567408.
7. Ponikowski P, Voors AA, Anker SD, et al. 2016 ESC Guidelines for the diagnosis and treatment of acute and chronic heart failure: The Task Force for the diagnosis and treatment of acute and chronic heart failure of the European Society of Cardiology (ESC) Developed with the special contribution of the Heart Failure Association (HFA) of the ESC. Eur Heart J. 2016; 37(27): 2129-2200, doi: 10.1093/eurhearti/ehw128, indexed in Pubmed: 27206819.

8. Yancy C, Jessup M, Bozkurt B, et al. 2017 ACC/AHA/HFSA Focused Update of the 2013 ACCF/AHA Guideline for the Management of Heart Failure: A Report of the American College of Cardiology/American Heart Association Task Force on Clinical Practice Guidelines and the Heart Failure Society of America. Circulation. 2017; 136(6): e137-e161, doi: 10.1161/ cir.0000000000000509.

9. Joglar JA, Acusta AP, Shusterman NH, et al. Effect of carvedilol on survival and hemodynamics in patients with atrial fibrillation and left ventricular dysfunction: retrospective analysis of the US Carvedilol Heart Failure Trials Program. Am Heart J. 2001; 142(3): 498-501, doi: 10.1067/mhj.2001.117318, indexed in Pubmed: 11526364.

10. Lechat P, Hulot JS, Escolano S, et al. Heart rate and cardiac rhythm relationships with bisoprolol benefit in chronic heart failure in CIBIS II Trial. Circulation. 2001; 103(10): 1428-1433, indexed in Pubmed: 11245648.

11. Mulder BA, van Veldhuisen DJ, Crijns HJ, et al. Effect of nebivolol on outcome in elderly patients with heart failure and atrial fibrillation: insights from SENIORS. Eur J Heart Fail. 2012; 14(10): 1171-1178, doi: 10.1093/eurjhf/hfs100, indexed in Pubmed: 22764183.

12. van Veldhuisen DJ, Aass H, El Allaf D, et al. MERIT-HF Study Group. Presence and development of atrial fibrillation in chronic heart failure. Experiences from the MERIT-HF Study. Eur J Heart Fail. 2006; 8(5): 539-546, doi: 10.1016/j.ejheart.2006.01.015, indexed in Pubmed: 16567126.

13. Kao DP, Davis G, Aleong R, et al. Effect of bucindolol on heart failure outcomes and heart rate response in patients with reduced ejection fraction heart failure and atrial fibrillation. Eur J Heart Fail. 2013; 15(3): 324-333, doi: 10.1093/eurjhf/hfs181, indexed in Pubmed: 23223178.

14. Rienstra M, Damman K, Mulder BA, et al. Beta-blockers and outcome in heart failure and atrial fibrillation: a meta-analysis. JACC Heart Fail. 2013; 1(1): 21-28, doi: 10.1016/j.jchf.2012.09.002, indexed in Pubmed: 24621795.

15. Kotecha D, Holmes J, Krum H, et al. Efficacy of $\beta$ blockers in patients with heart failure plus atrial fibrillation: an individual-patient data meta-analysis. Lancet. 2014; 384(9961): 2235-2243, doi: 10.1016/S0140-6736(14)61373-8, indexed in Pubmed: 25193873 .

16. Nielsen $\mathrm{PB}$, Larsen TB, Gorst-Rasmussen $\mathrm{A}$, et al. $\beta$-Blockers in atrial fibrillation patients with or without heart failure: association with mortality in a nationwide cohort study. Circ Heart Fail. 2016; 9(2): e002597, doi: 10.1161/CIRCHEARTFAILURE.115.002597, indexed in Pubmed: 26823497.

17. Cadrin-Tourigny J, Shohoudi A, Roy D, et al. Decreased Mortality With Beta-Blockers in Patients With Heart Failure and Coexisting Atrial Fibrillation: An AF-CHF Substudy. JACC Heart Fail. 2017; 5(2): 99-106, doi: 10.1016/j.jchf.2016.10.015, indexed in Pubmed: 28089316.

18. Li SJ, Sartipy U, Lund LH, et al. Prognostic Significance of Resting Heart Rate and Use of $\beta$-Blockers in Atrial Fibrillation 
and Sinus Rhythm in Patients With Heart Failure and Reduced Ejection Fraction: Findings From the Swedish Heart Failure Registry. Circ Heart Fail. 2015; 8(5): 871-879, doi: 10.1161/CIRCHEARTFAILURE.115.002285, indexed in Pubmed: 26243796.

19. Jadad AR, Moore RA, Carroll D, et al. Assessing the quality of reports of randomized clinical trials: is blinding necessary? Control Clin Trials. 1996; 17(1): 1-12, indexed in Pubmed: 8721797.

20. Scheen AJ, Ernest P, Jandrain B. [How I explore ... a risk difference in the occurrence of an event in clinical trials]. Rev Med Liege. 2012; 67(11): 597-602, indexed in Pubmed: 23346831.

21. Pan An, Sun Qi, Okereke OI, et al. Depression and risk of stroke morbidity and mortality: a meta-analysis and systematic review. JAMA. 2011; 306(11): 1241-1249, doi: 10.1001/jama.2011.1282, indexed in Pubmed: 21934057.

22. Higgins JPT, Thompson SG. Quantifying heterogeneity in a meta-analysis. Stat Med. 2002; 21(11): 1539-1558, doi: 10.1002/ sim.1186, indexed in Pubmed: 12111919.

23. DerSimonian R, Laird N. Meta-analysis in clinical trials revisited. Contemp Clin Trials. 2015; 45(Pt A): 139-145, doi: 10.1016/j. cct.2015.09.002, indexed in Pubmed: 26343745.

24. Simpson J, Castagno D, Doughty RN, et al. Is heart rate a risk marker in patients with chronic heart failure and concomitant atrial fibrillation? Results from the MAGGIC meta-analysis. Eur
J Heart Fail. 2015; 17(11): 1182-1191, doi: 10.1002/ejhf.346, indexed in Pubmed: 26358762.

25. Wan H, Yang Y, Zhu J, et al. Prognostic value of ventricular heart rate in patients with permanent atrial fibrillation and heart failure. Int J Cardiol. 2015; 182: 70-71, doi: 10.1016/j.ijcard.2014.12.120, indexed in Pubmed: 25576723.

26. Cullington D, Goode KM, Zhang J, et al. Is heart rate important for patients with heart failure in atrial fibrillation? JACC Heart Fail. 2014; 2(3): 213-220, doi: 10.1016/j.jchf.2014.01.005, indexed in Pubmed: 24952686.

27. Miller RJH, Howlett JG, Chiu MH, et al. Relationships among achieved heart rate, $\beta$-blocker dose and long-term outcomes in patients with heart failure with atrial fibrillation. Open Heart. 2016; 3(2): e000520, doi: 10.1136/openhrt-2016-000520, indexed in Pubmed: 28123760.

28. Groenveld HF, Tijssen JGP, Crijns HJ, et al. Lenient versus strict rate control in patients with atrial fibrillation. $\mathrm{N}$ Engl J Med. 2010; 362(15): 1363-1373, doi: 10.1056/NEJMoa1001337, indexed in Pubmed: 20231232.

29. Mulder BA, Van Veldhuisen DJ, Crijns HJ, et al. Lenient vs. strict rate control in patients with atrial fibrillation and heart failure: a post-hoc analysis of the RACE II study. Eur J Heart Fail. 2013; 15(11): 1311-1318, doi: 10.1093/eurjhf/hft093, indexed in Pubmed: 23759284. 